\title{
Constraints on variation of weight-shifting by foot during walking in adolescents with valgus alignment of hallux
}

\author{
EWA LATOUR $^{1 *}$, JAROSŁAW ARLET ${ }^{1}$, MAREK LATOUR $^{2}$, \\ LECHOSŁAW B. DWORAK ${ }^{3}$, ANDRZEJ BOHATYREWICZ ${ }^{4}$ \\ ${ }^{1}$ Poznan University of Physical Education, Faculty of Physical Culture in Gorzów Wielkopolski, Poznań, Poland. \\ ${ }^{2}$ Multidisciplinary Regional Hospital, Department of Orthopaedics, Gorzów Wielkopolski, Poland. \\ ${ }^{3}$ Higher Vocational State School in Kalisz, Faculty of Physiotherapy, Kalisz, Poland. \\ ${ }^{4}$ Department of Orthopaedics Traumatology and Musculoskeletal Oncology, Pomeranian Medical University, \\ Szczecin, Poland.
}

\begin{abstract}
Purpose: Etiology of hallux valgus (HV) remains unclear and effective treatments and prophylaxis for this condition are lacking and conclusions of researches concerning HV are inconsistent. Recognition of the function-structure interrelation in foot at the early stage of valgus alignment of first metatarsophalangeus joint (1stMTPJ) would help explain the etiology of HV. Methods: The frequency of weight-shifting patterns during walking and the body mass index (BMI) were assessed relative to goniometric measurements of the 1stMTPJ angle in adolescents. Weight-shifting patterns were identified with a plantar pressure analysis, performed with the GaitlineMaxline Distance method. Results: As the 1stMTPJ angle widened, the frequency distributions of identified weight-shifting patterns became increasingly lopsided, due to the favoring or disfavoring of one pattern at the expense of the other. The two sexes showed opposite trends in the weight-shifting patterns that were favored/disfavored, relative to participants with a $0^{\circ} 1$ stMTPJ angle. The clear predominance of a central-shift pattern, at the expense of a medial-shift, occurred with the largest $\left(20^{\circ}\right)$ 1stMTPJ angle among girls. Additionally, the BMI distributions associated with 1stMTPJ angle characteristics showed opposite trends between the sexes. Conclusions: Valgus alignment of 1stMTPJ among adolescents were associated with limitations in weight-shifting freedom of the foot during walking, that could be involved in maintaining balance. An 1stMTPJ angle of $20^{\circ}$, where distinct limitations were observed, might serve as a criterion of HV pathology.
\end{abstract}

Key words: walking, body mass index, motor control, hallux valgus

\section{Introduction}

The valgus alignment of the first metatarsophalangeal joint (1stMTPJ) is a feature perceived as a risk factor for developing the pathology called "hallux valgus" (HV) [25]. This troublesome deformation of the foot [5], typically begins in adolescence [25], develops with age and increases a fall risk in older individuals [22]. The etiology and pathophysiology of HV are not fully understood, moreover, there is a lack of effective treatments and prophylaxis for this condition [12]. Therefore there is a need to investigate functional problems related to different structural states of hallux and new foot assessment methods. Useful information about foot function during walking comes from studies using pedobarography, but they provide inconsistent conclusions [4], [14], [24]. Some studies proposed that $\mathrm{HV}$ arose from a lateral load transfer that resulted from a reduction in the weight-bearing function of the medial toe [20], which suggested a supination-based mechanism. Others proposed the opposite load distribution, which suggested a pronation-based distribution [24]. Still other studies could not confirm a relationship between hallux deformities and plantar pressure distributions [14], [29]. In addition, earlier research mainly targeted valgus states in adults that were perceived as pathological, consequently, it was

\footnotetext{
* Corresponding author: Ewa Latour, Department of Physiotherapy, Poznan University of Physical Education, Faculty of Physical Culture in Gorzów Wielkopolski, ul. Estkowskiego 13, 66-400, Gorzów Wielkopolski, Poland. E-mail: ewalatour@tlen.pl

Received: April 16th, 2020

Accepted for publication: June 15th, 2020
} 
difficult to identify a structure-function relationship that might lead to the development of the deformation.

The need to clarify the etiology of HV inspired this investigation of functional phenomena related to hallux alignment in healthy individuals, during the period of life when only poorly expressed valgus alignment (not yet classified as HV) could potentially be observed [25]. This study assumed that, if there are specific structure-function relationships that underlie the development of $\mathrm{HV}$, distinct functional foot features that reflect the degree of valgus alignment in the 1st MTPJ should be identifiable before $\mathrm{HV}$ is diagnosed.

The objective of this study was to identify the early functional expression of a valgus alignment in the hallux in an unselected group of healthy adolescents based on analyzing how differences in plantar pressure distributions during walking were related to the angle of 1 stMTPJ alignment.

\section{Materials and methods}

\subsection{Participants}

A group of 60 adolescents ( 33 girls and 27 boys), was recruited for this study. The inclusion criteria were: age from 11 to 12 years, written informed consent to participate in the study, and approval by parent or legal guardians. The exclusion criteria were diseases limiting the efficiency of movement. All participants were pupils from the same grade level of a public school. The representativeness of the study group was verified, based on a comparison with centile grids, prepared with 1000-people groups of girls and boys of the same age from the same city. The mean (standard deviation) values of body weight, body height, and BMI, respectively, were: $42.15(\mathrm{SD}=9.39) \mathrm{kg}, 1.53$ $(\mathrm{SD}=0.09) \mathrm{m}, 17.91(\mathrm{SD}=2.96) \mathrm{kg} / \mathrm{m}^{2}$ for girls and $43.19(\mathrm{SD}=10.65) \mathrm{kg}, 1.53(\mathrm{SD}=0.08) \mathrm{m}, 18.12$ $(\mathrm{SD}=3.01) \mathrm{kg} / \mathrm{m}^{2}$ for boys. This study was approved by the Medical Academy's Bioethics Committee in Poznań, Poland.

\subsection{Measurement methods}

The assessment included the weight-shifting by the foot during walking, body structure described by body mass index (BMI) and the relationship between these parameters and goniometric measurements of 1st MTPJ alignment.
Assessment of 1st MTPJ alignment was obtained by measuring the angle (1stMTPJA) formed by the longitudinal axes of two joint bones: the first metatarsal bone and the proximal phalanx of the hallux. All measurements were performed in the transverse plane, with the goniometric method, because it was the simplest, most traditional, most widely available and well described method [17]. This procedure did not require $\mathrm{X}$-ray imaging, consequently, data collection was lowrisk and non-invasive. The measurements were performed with the participant lying in the supine position, with an accuracy of $5^{\circ}$. This accuracy and maximum measured value allowed to adopt the following categories of 1stMTPJA: $0^{\circ}$, for results $\left\{0^{\circ}, 5^{\circ}\right\}$ as a zero-1stMTPJA reference group, and non-zero-1 st MTPJA groups: $10^{\circ}, 15^{\circ}$, and $20^{\circ}$, respectively. The reliability of goniometric assessments estimated with the Kendall correlation coefficient for two series of measurements was $\tau=0.55$ (approximately 0.76 in a Spearman correlation scale).

Assessment of body weight shifting through the plantar surface of the foot during walking, conducted according to the second step protocol [12], was based on measurement results from a pedobarographic platform (PEL 38; Medicapteurs, Toulouse, France) with an active area of $320 \times 320 \mathrm{~mm}$, sensor dimensions of $10 \times$ $10 \mathrm{~mm}$, and a 5-m walkway. Reliability assessment of the platform, caried out using the intraclass correlation (ICC) method for model block measurements, presented 0.979 of consistency and 0.961 of agreement.

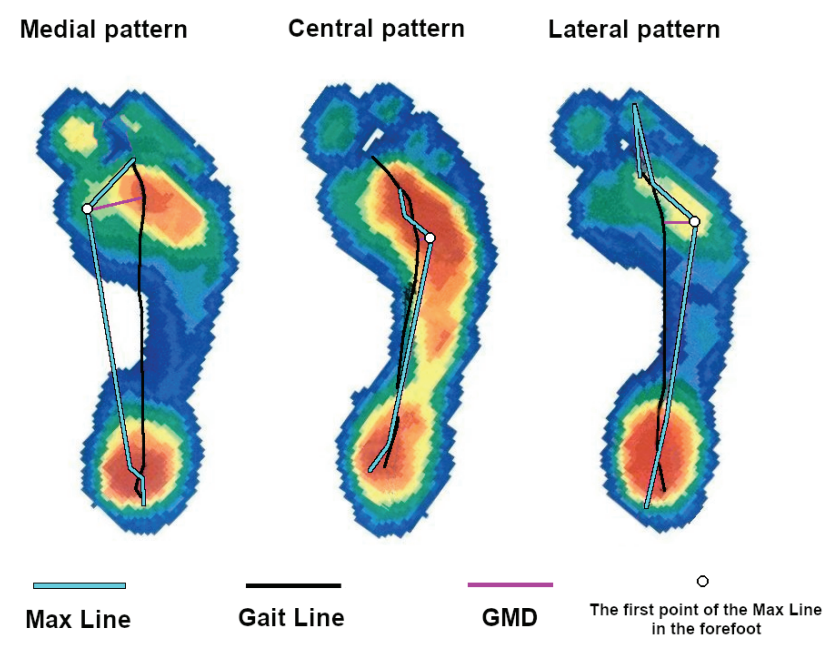

Fig. 1. Illustration of measurements representing three weight-shift patterns: medial, central, and lateral. Abbreviations: Gait Line $=$ a line connecting centroids of pressures registered in subsequent moments of measurement; Max Line $=\mathrm{a}$ line connecting the positions of pressures of the maximal value, recorded at subsequent moments of measurement; Gaitline-Maxline Distance (GMD) = the shortest distance between the first point of the Max Line in the forefoot and Gait Line 
Each of the measurements was characterized with one among three patterns of weight-shifting: medial, central and lateral (Fig. 1) by the Gaitline-Maxline Distance (GMD) method [13], identified on the basis of cluster analysis of the spatial distance in the forefoot between two spatiotemporal indices of plantar pressure distribution: the Gaitline, which represented the progression of the center of pressure and the Maxline, which represented the progression of the peak pressure.

The total number of measurements carried out in six trials for each participant - three for each foot was 360 , i.e., $33 \cdot 6=198$ in girls and $27 \cdot 6=62$ in boys.

\subsection{Statistical analysis}

For each participant, the individual tendency to use a particular weight-shifting pattern, estimated as the relative frequencies of weight-shifting patterns (RFSP), was calculated as the percentage of steps performed according to a specific pattern among the total number of steps performed by one participant.

To determine how the RFSPs were affected by sex, estimation of differences in RFSPs and evaluation of dissimilarity between girls and boys were carried out. These evaluations were performed in all participants, in participants with zero-1stMTPJAs, and in participants with non-zero-1 stMTPJAs.

To assess the functional effect of the hallux alignment, the strength of dissimilarity (Cramer's effect size $-V$ ) was estimated between sets of RFSPs for participants with non-zero-1stMTPJAs and participants with zero-1stMTPJAs. Also, the differences between corresponding RFSPs in sets ( $\triangle \mathrm{RFSP}$ ) were calculated, as follows:

$$
\Delta \mathrm{RFSP}=\mathrm{RFSP}_{\text {non-zero-1stTMPJA }}-\mathrm{RFSP}_{\text {zero-1stMTJA }}
$$

for each sex separately.

Statistical analyses and charts were performed and developed with the R Language and Environment for Statistical Computing. The strengths of dissimilarity between the sets of RFSPs determined for particular 1stMTPJA subgroups were assessed with the Cramer's effect size $(V)$ [21]. The differences between RFSPs are expressed with 95\% confidence intervals (CIs), the borders of which are presented in square brackets $\left(\left[\mathrm{CI}_{\text {lower }} \mathrm{CI}_{\text {upper }}\right]\right)$. The confidence interval borders for calculated proportions were estimated with the function of Blaker's exact CI for a binomial proportion [3]. Differences between two proportions were calculated with the Wald interval function ("wald2ci" from package "PropCIs") [1]. The strength of differ- ences in BMI values between 1stMTPJA subgroups was calculated with Cliff's effect size $(\delta)[6]$.

\section{Results}

The goniometric measurements (Fig. 2) showed 1stMTPJA values of 0 to $20^{\circ}$. Girls were more likely than boys to exhibit a valgus alignment at the 1stMTPJ.

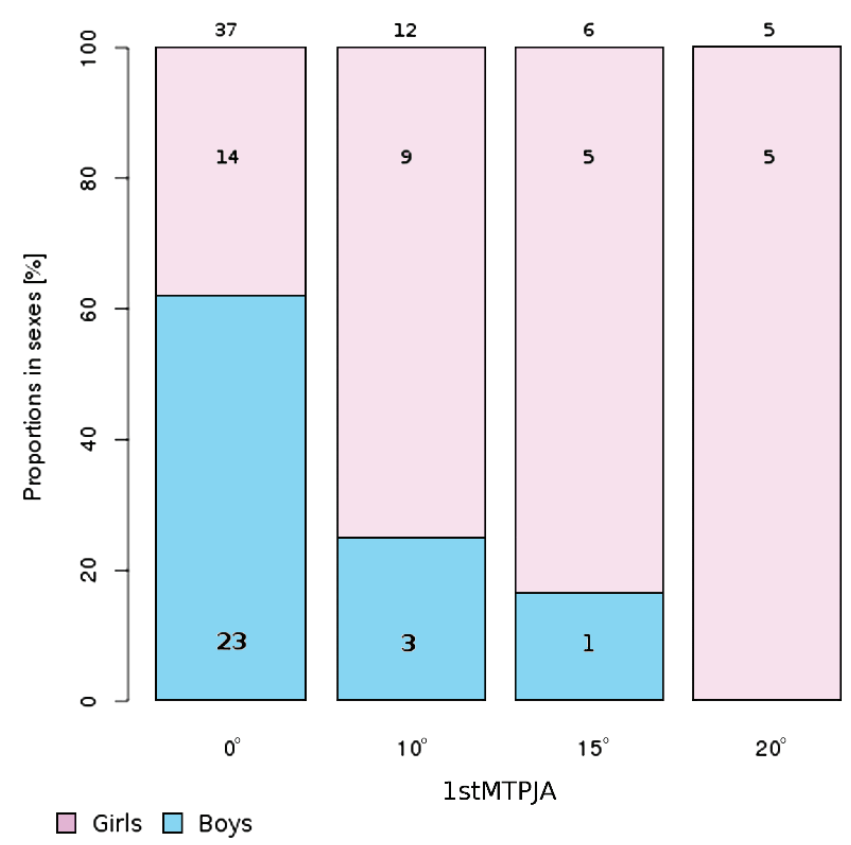

Fig. 2. Sex proportion in terms of 1stMTPJA in studied group. The proportion of girls (light gray) increases as the 1stMTPJA increases. The data in each field and above the bars denotes number of participants.

1stMTPJA = first metatarsophalangeal joint angle

A preliminary analysis of the differences in RFSPs between boys and girls, conducted for all participants, for participants with zero 1stMTPJAs, and for those with non-zero 1stMTPJAs (Fig. 3, Table 1), revealed that boys exhibited the central pattern more frequently than girls, independently of the 1stMTPJA. In contrast, the frequencies of lateral and medial patterns among the sexes depended on the 1stMTPJA. In the zero-1stMTPJA group, boys used the medial pattern less frequently and the lateral pattern more frequently than girls. Conversely, in the non-zero-1stMTPJA group, boys used the medial pattern more frequently and the lateral pattern less frequently than girls. Consequently, participant sex was taken into account in further analyses.

The comparison between RFSPs at individuals with non-zero-1stMTPJAs and those with zero-1stMTPJAs revealed that sets of RFSPs in the non-zero-1stMTPJA 

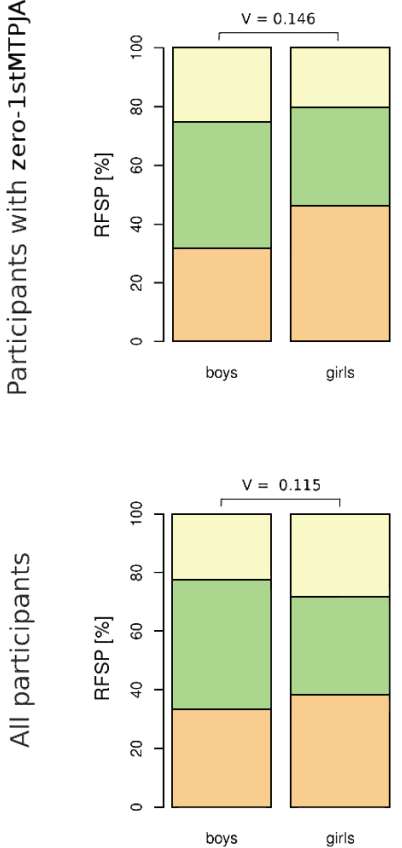

$\square$ Lateral

$\square$ Central

$\square$ Medial

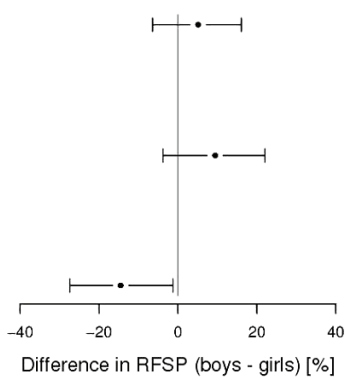

$\square$ Lateral

$\square$ Central

$\square$ Medial

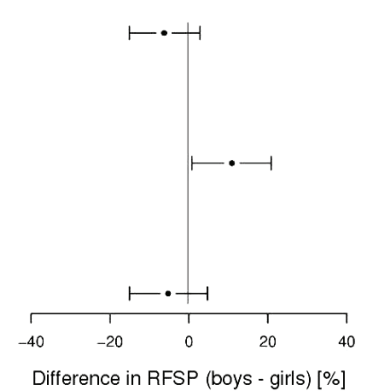

Difference in RFSP (boys - girls) [\%]

$\square$ Lateral

$\square$ Central

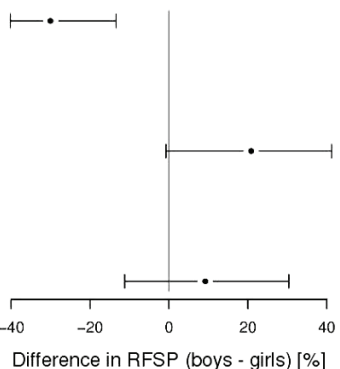

Fig. 3. Comparison of RFSPs between boys and girls, based on 1stMTPJ structure. Bar graphs show that sex affected the frequency of shift patterns: the central pattern was more common in boys than in girls, independent of 1stMTPJA. The sex preponderance of medial and lateral pattern frequencies depended on the 1stMTPJA.

Line graphs show sex differences in RFSPs (boys - girls) for the three patterns with $95 \%$ confidence intervals.

Abbreviations: $V$ - the Cramer's effect size,

1 stMTPJA - first metatarsophalangeal joint angle, RFSP - relative frequency of weight-shifting patterns

Table 1. Comparison of RFSPs between boys and girls, based on 1stMTPJ structure

\begin{tabular}{|c|c|c|c|c|c|}
\hline & & & RFSP & & \\
\hline 1stMTPJA & Patterns & Boys & Girls & Boys - Girls & Cramer's $V$ \\
\hline \multirow{3}{*}{ Zero $\left(0^{\circ}\right)$} & Lateral & 25.36 & 20.24 & 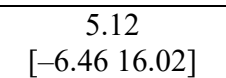 & \multirow{3}{*}{0.146} \\
\hline & Central & 42.75 & 33.33 & $\begin{array}{c}9.42 \\
{[-3.7922 .06]}\end{array}$ & \\
\hline & Medial & 31.88 & 46.43 & $\begin{array}{c}-14.54 \\
{[-27.44-1.29]}\end{array}$ & \\
\hline \multirow{3}{*}{ All $\left(\geq 0^{\circ}\right)$} & Lateral & 22.22 & 28.28 & $\begin{array}{c}-6.06 \\
{[-14.893 .01]}\end{array}$ & \multirow{3}{*}{0.115} \\
\hline & Central & 44.44 & 33.33 & $\begin{array}{c}11.11 \\
{[0.9821 .04]}\end{array}$ & \\
\hline & Medial & 33.33 & 38.38 & 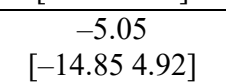 & \\
\hline \multirow{3}{*}{ Non-zero $\left(>0^{\circ}\right)$} & Lateral & 4.17 & 34.21 & $\begin{array}{c}-30.04 \\
{[-40.20-13.38]}\end{array}$ & \multirow{3}{*}{0.255} \\
\hline & Central & 54.17 & 33.33 & $\begin{array}{c}20.83 \\
{[-0.7841 .23]}\end{array}$ & \\
\hline & Medial & 41.67 & 32.46 & $\begin{array}{c}9.21 \\
{[-11.2730 .37]}\end{array}$ & \\
\hline
\end{tabular}

Abbreviations: 1stMTPJ - hallux metatarsophalangeal joint, 1stMTPJA - 1stMTPJ angle, RFSP - relative frequency of weight-shifting patterns, Cramer's $V$ - effect size of association between sets of RFSP for boys and girls. Numbers in square brackets denote the $95 \%$ confidence interval of the value shown above them. 
Table 2. Comparison of RFSPs between individuals with non-zero-1stMTPJA and those with zero-1stMTPJA

\begin{tabular}{|c|c|c|c|c|c|c|}
\hline & & & & $1 \mathrm{stl}$ & PJA & \\
\hline Sex & Patterns & Quantity & $0^{\circ}$ & $10^{\circ}$ & $15^{\circ}$ & $20^{\circ}$ \\
\hline \multirow{8}{*}{ Girls } & \multirow[b]{2}{*}{ Lateral } & RFSP & 20.24 & 33.33 & 40.00 & 30 \\
\hline & & $\triangle \mathrm{RFSP}$ & $\times$ & $\begin{array}{c}13.10 \\
{[-2.0928 .09]}\end{array}$ & $\begin{array}{c}19.76 \\
{[0.6338 .76]}\end{array}$ & $\begin{array}{c}9.76 \\
{[-7.9028 .54]}\end{array}$ \\
\hline & \multirow[b]{2}{*}{ Central } & RFSP & 33.33 & 29.63 & 13.33 & 60 \\
\hline & & $\triangle \mathrm{RFSP}$ & $\times$ & $\begin{array}{c}-3.70 \\
{[-19.0112 .28]}\end{array}$ & $\begin{array}{c}-20.00 \\
{[-34.16-2.03]}\end{array}$ & $\begin{array}{c}26.67 \\
{[5.9245 .39]}\end{array}$ \\
\hline & \multirow{4}{*}{ Medial } & RFSP & 46.43 & 37.04 & 46.67 & 10 \\
\hline & & $\triangle \mathrm{RFSP}$ & $\times$ & $\begin{array}{c}-9.39 \\
{[-25.507 .48]}\end{array}$ & $\begin{array}{c}0.24 \\
{[-19.8920 .61]}\end{array}$ & $\begin{array}{c}-36.43 \\
{[-49.58-18.44]}\end{array}$ \\
\hline & & Cramer's $V$ & $x$ & 0.15 & 0.274 & 0.406 \\
\hline & & BMI & $\begin{array}{c}17.78 \\
{[16.1620 .14]} \\
\end{array}$ & $\begin{array}{c}18.96 \\
{[14.0119 .7]} \\
\end{array}$ & $\begin{array}{c}19.04 \\
{[16.8821 .21]} \\
\end{array}$ & $\begin{array}{c}16.14 \\
{[13.9117 .83]} \\
\end{array}$ \\
\hline \multirow{8}{*}{ Boys } & \multirow[b]{2}{*}{ Lateral } & RFSP & 25.36 & 0.00 & 16.67 & $x$ \\
\hline & & $\triangle \mathrm{RFSP}$ & $\times$ & $\begin{array}{c}-25.36 \\
{[-32.70-8.73]}\end{array}$ & $\begin{array}{c}-8.70 \\
{[-31.5830 .15]}\end{array}$ & $x$ \\
\hline & \multirow[b]{2}{*}{ Central } & RFSP & 42.75 & 66.67 & 16.67 & $x$ \\
\hline & & $\triangle \mathrm{RFSP}$ & $\times$ & $\begin{array}{c}23.91 \\
{[-0.3144 .60]}\end{array}$ & 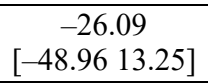 & $x$ \\
\hline & \multirow{4}{*}{ Medial } & RFSP & 31.88 & 33.33 & 66.67 & $x$ \\
\hline & & $\triangle \mathrm{RFSP}$ & $\times$ & $\begin{array}{c}1.45 \\
{[-19.4325 .15]}\end{array}$ & $\begin{array}{c}34.78 \\
{[-4.0764 .78]}\end{array}$ & $\times$ \\
\hline & & Cramer's $V$ & $\times$ & 0.691 & 0.357 & $x$ \\
\hline & & BMI & $\begin{array}{c}17.83 \\
{[16.2519 .61]}\end{array}$ & $\begin{array}{c}16.2 \\
{[15.5617 .51]}\end{array}$ & 16.22 & $x$ \\
\hline
\end{tabular}

Abbreviations: 1stMTPJA - hallux metatarsophalangeal joint angle, RFSP - relative frequency of weight-shifting patterns, $\triangle$ RFSP - difference between RFSP for participants with non-zero 1stMTPJA and participants with zero-1 stMTPJA, Cramer's $V$ - effect size of association between sets of RFSP for participants with non-zero 1stMTPJA and participants with zero-1stMTPJA. Numbers in square brackets denote the $95 \%$ confidence interval of the value shown above them.

group became increasingly more distinct from the sets in the zero-1stMTPJA group, as the 1stMTPJA increased. Thus, a particularly high absolute $\triangle$ RFSP value presented participants with a $20^{\circ} 1$ stMTPJA (Fig. 4A, Table 2). In that group, the distribution of RFSPs was lopsided, in terms of favoring or disfavoring one RFSP at the expense of another.

Moreover, the distribution of $\triangle \mathrm{RFSPs}$ varied according to sex (Fig. 4B). For girls, when the 1stMTPJA increased, the frequency of the lateral pattern also increased. For $10^{\circ}$ and $15^{\circ} 1$ stMTPJAs, when the lateral pattern frequency increased, the medial and central patterns decreased, respectively. For $20^{\circ} 1$ stMTPJAs, both the lateral and central patterns increased at the expense of the medial pattern. However, for boys, the RFSPs changed in the opposite directions, which created nearly a mirror image to the RFSPs for girls. Boys with non-zero 1stMTPJAs were characterized by a lower frequency of the central pattern and a higher frequency of the medial pattern, compared to boys with zero 1stMTPJAs.

BMI values varied with 1stMTPJA values. The relationships between BMIs and 1stMTPJA values also depended on sex (Fig. 5). For participants with zero-1 stMTPJA values, the BMIs were similar for both the sexes. However, for intermediate 1stMTPJA values, the relationship between the BMI and the 1stMTPJA depended on sex: in the non-zero-1stMTPJA groups, girls had higher BMIs and boys had lower BMIs, compared to the corresponding zero-1stMTPJA groups. In the highest 1stMTPJA groups, the BMIs were similar and lowest, for the two sexes.

\section{Discussion}

This study characterized the relationship between foot function and 1stMTPJ alignment in adolescents. The essential result was that as the 1stMTPJA grew larger, distributions of relative frequencies of shift patterns (RFSP) became increasingly different from the distributions observed with zero-1stMTPJA. These differences in RFSPs reflected limitations in the free use of weight-shifting patterns. Thus, persons with severe 1stMTPJAs had to rely predominantly on a single 
A
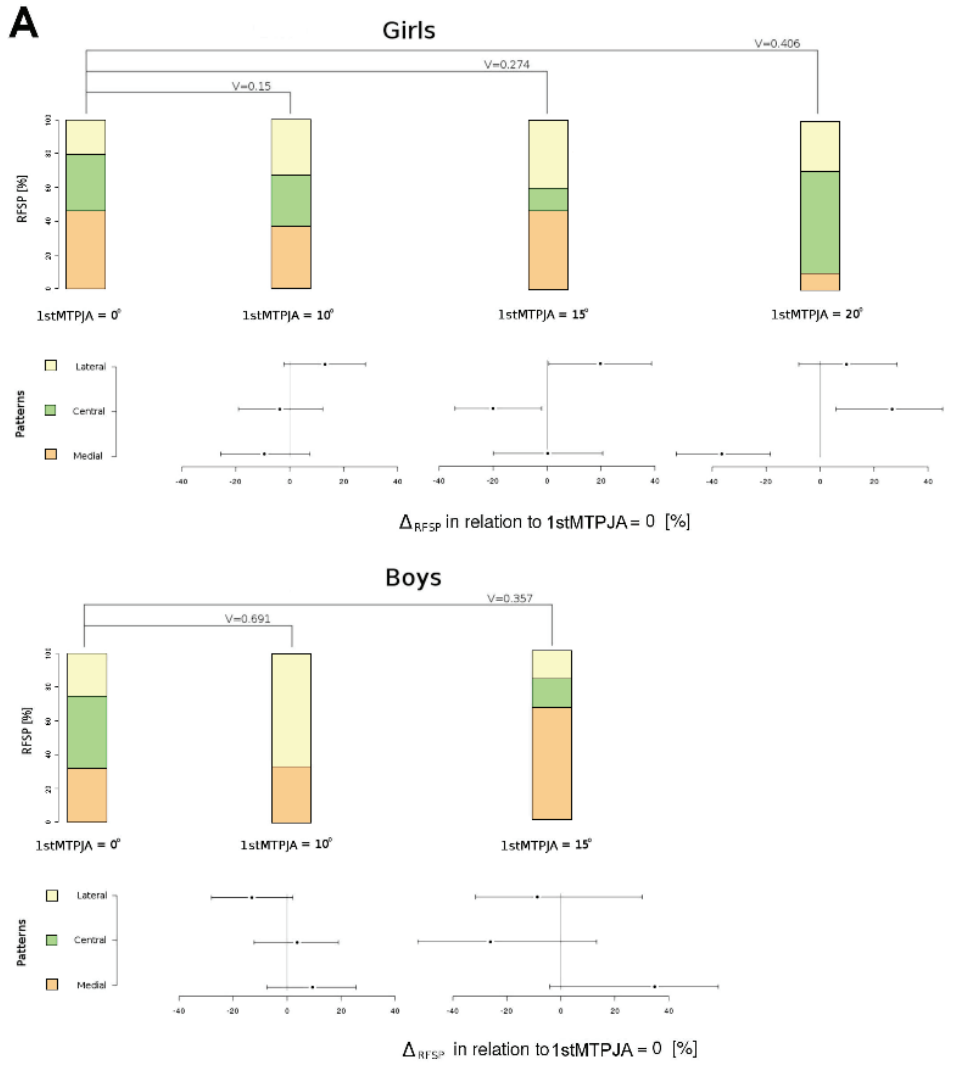

B
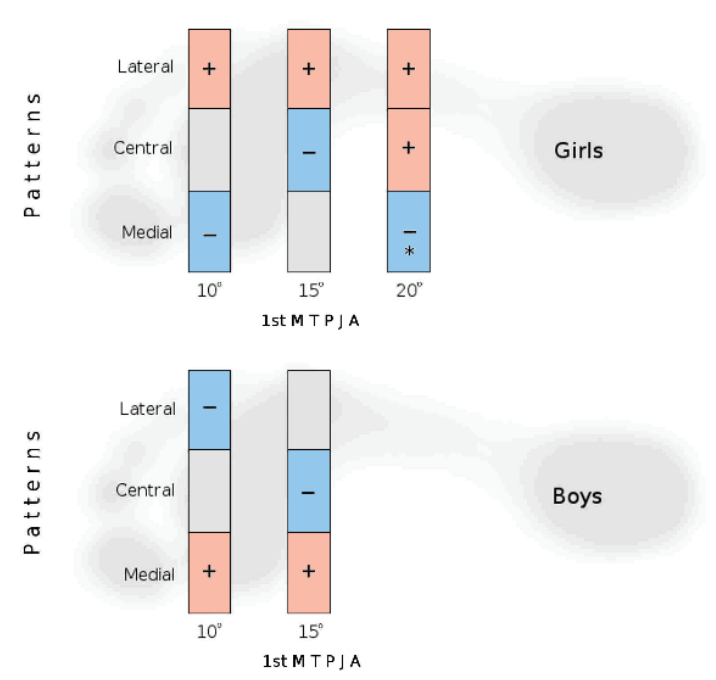

shift-pattern, when the feet performed weight-shifting. Indeed, for girls with the largest 1 stMTPJAs $\left(20^{\circ}\right)$, the RFSP distribution represented considerable limitations in using the medial shifting pattern, and the central pattern was preferred. This information was supplemented by the mirror-image sex-related inversion of 1stMTPJA characteristics associated with both the RFSP (Fig. 4) and the BMI (Fig. 5). Correspondence in these results taken together indicated that they were reliable, despite the relatively small sample sizes in the subgroups of patients with 1stMTPJA values greater than $0^{\circ}$.
Fig. 4. Comparisons of RFSPs between individuals with non-zero-1stMTPJA and those with zero-1stMTPJA (bar graphs). The $\triangle$ RFSP, for girls is more observable with growth of 1stMTPJA value. $\triangle$ RFSP is shown with $95 \%$ confidence intervals (line graphs). The simplified drawings (B) comprise a qualitative representation of $\triangle$ RFSP shown in A.

Red areas marked with $(+)$ and blue areas marked with (-) indicate that the absolute value of $\triangle \mathrm{RFS}$ was greater than $3 / 4$ the margin of error. Grey areas indicate that the difference described by $\triangle$ RFSP was not clearly observable. The asterisk denotes that the difference was distinctly evident.

The relative differences changed in opposite directions between girls and boys, resulting in almost mirror images. Abbreviations: 1stMTPJA - first metatarsophalangeal joint angle, RFSP - relative frequency of weight-shifting patterns, $\triangle$ RFSP - difference between RFSP for participants with non-zero 1stMTPJA and participants with zero-1stMTPJA, $V$ - the Cramer's effect size
Motor control theories [18] indicate that individuals naturally use a variety of shift patterns to shift the weight through the plantar surface while walking. This property is hereinafter referred to as "weight-shifting freedom". The results from analyzing the structurefunction relationship revealed that structural features mediated this natural variety in RFSPs by shaping the motion strategy, under the general rule of functionally freezing the degrees of freedom [26], [28]. Thus, favoring one shift-pattern over the other patterns meant a limitation in weight-shifting freedom. This limitation was expressed most noticeably with the largest 
alignment deflections, even in a group of adolescents with generally small physiological valgus alignments. Consequently, this limitation should be considered a factor in HV etiology.

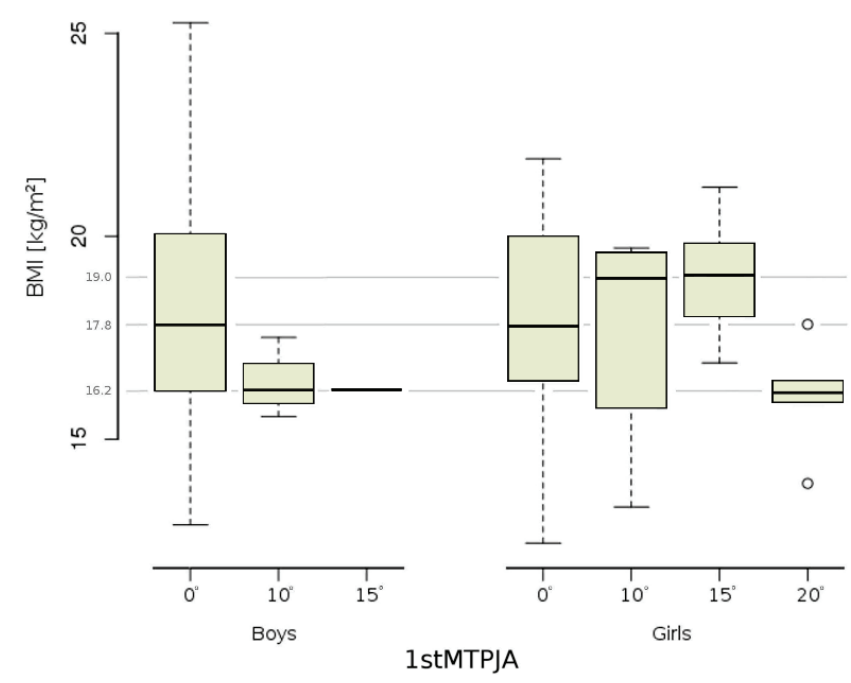

Fig. 5. 1stMTPJA characteristics of BMI for both sexes. The differences between subgroups were evaluated with Cliff's effect size $(\delta)(9)$. Boys and girls with zero-1 stMTPJA had similar BMI values $(\delta=0.006)$. Boys and girls with 1stMTPJA of $10-15^{\circ}$ showed opposite trends in the association between 1stMTPJA and BMI $(\delta=0.41)$ compared to the zero-1stMTPJA. However, girls and boys with the most extreme 1stMTPJA values had similar $(\delta=0.19)$ BMI values, and these BMIs were the lowest in the study groups (for girls $\delta=0.54$, for boys $\delta=0.33$, with respect to BMIs associated with zero-1stMTPJA)

The observation of a predominantly high level of weight-shifting freedom in the study group was consistent with the earlier hypothesis that variability in the human motor system is the pervasive "healthy norm" [26]. The adaptability and flexibility of a dynamic system are expressed as a multiplicity of modes of action [2], consequently, weight-shifting freedom appears to be a desired feature for gait efficiency. The purpose of the foot is to perform propulsion while maintaining balance; thus, the foot requires freedom of motion to adapt to various terrains. Therefore, the limitation in weight-shifting freedom featured in the valgus alignment of the hallux might reduce an individual's environmental adaptability. In particular, advanced deformations can lead to gait instability and falling susceptibility, as reported in studies on HV [22]. Indeed, studies have shown that HV and fall risk converged with reduced gait stability, and this convergence was reflected in the increased intra-individual variability in time parameters [15]. However, no noticeable changes in intra-individual variability were reported in the plantar pressure distributions of the forefoot, mid-foot, or rear-foot areas, either in older individuals [9] or in individuals with HV [12]. Insufficient gait stability in advanced $\mathrm{HV}$ states might be explained by revealed in the study limitation in foot motor behavior featuring valgus structure. This finding supports the need of taking into account the phenomenon of movement variability to a greater extent in studies on $\mathrm{HV}$ and fall risk.

The comparison of $\triangle$ RFSPs (Fig. 4) revealed also their mirror-image sex-relation. This suggested that a valgus alignment involves different, sex-related foot movement strategies, characterized by relatively higher incidences of the lateral-shift pattern in girls and the medial-shift pattern in boys. In turn, this hypothesis suggested that valgus formations of the hallux develop along two different, sex-specific pathways. Additionally, differences in sex characteristics of pattern proportions have also been disclosed: boys more frequently used the central-shift pattern compared to girls (Fig. 3), which is consistent with the earlier studies on healthy individuals in this age group [27]. These sexrelated specificities in hallux structure-function effects provide a rationale for performing gait analyses separately for each sex, as it was done in some previous studies on HV [11], [16], [23].

Participants with valgus hallux alignment (non-zero-1 stMTPJA) represented a relatively large part of the study group, particularly among girls, consistent with the known female preponderance in the HV incidence [23]. However, a relatively small group of girls had the largest deflections $\left(20^{\circ}\right)$, which suggested a pathological state. Indeed, this group showed the greatest dissimilarity in pattern frequencies with an exceptionally frequent use of the central-shift pattern, compared to the zero-1 stMTPJA group. In addition, the vast majority of participants had smaller 1stMTPJAs $\left(10\right.$ and $\left.15^{\circ}\right)$ and showed a much smaller degree of dissimilarity than participants with $20^{\circ} 1$ stMTPJAs, in terms of pattern frequencies, compared to the zero-1stMTPJA group. Thus, it is likely that the dissimilarities between the zero-1stMTPJA group and the $10^{\circ}$ and $15^{\circ}$ 1stMTPJA groups reflected different physiological ways of shifting weight in both pathways to valgus formation. Therefore, these dissimilarities should, at most, be considered only risk factors for $\mathrm{HV}$, which might not necessarily lead to pathology. These observations can be used as a starting point for research on the determination of diagnostic criteria that are currently lacking for HV pathology, and the angle at which a distinct functional sign is observed might serve as a diagnostic criterion for HV pathology.

Analogously to the weight-shifting patterns, the BMI distributions across 1stMTPJAs, displayed mirror-image 
sex-related effects. These two 1stMTPJA-related distributions led to the inference that the limitation in weight-shifting freedom in valgus alignment of the hallux might to be related to BMI. This suggests, that assuming that the BMI represents the whole-body structure, and thus, determines the general movement strategy for maintaining balance while performing a weight shift [13], the functional limitations of the foot might reflect a body-structure-determined strategy for maintaining balance [7].

The sex-related opposite BMI - 1stMTPJA associations also supported the hypothesis that there are two pathways for valgus alignment. This hypothesis was previously proposed in a study on BMIs in an older group (mean age of 78) with at least $15^{\circ} \mathrm{HV}$ [23], which hypothesized that the two different pathways to HV might be explained by sex-specific shoe choices. However, the construction of shoes for younger adolescents is determined much less by sex than shoes for adults. Thus, the BMI-determined strategy for maintaining balance during whole body movements could be an important factor in the development of hallux structure.

An exceptionally frequent use of the central-shift pattern among individuals with the largest hallux deflections (Fig. 4) was consistent with central overloading in the forefoot reported in two recent HV studies conducted in large groups of participants with disproportionate numbers of women $(95 \%$ of 679 HV patients [30] and $74 \%$ of $764 \mathrm{HV}$ patients [10]). Whereas deflection-size dependence of RFSP distributions in girls might explain inconsistent, often contradictory results presented by previous studies on HV-related loadings [24]; this could make a substantial impact on results, due to the known preponderance of women in $\mathrm{HV}$-studies. Girls exhibited a higher frequency of the lateral-shift pattern, which supported a supination HV etiology, irrespective of the size of deflection, even for very small deflections. Although a distinct reduction in the medial RFSP would also support this etiology, however that type of reduction only appeared with the largest deflection size. Previous analyses applied a $15^{\circ}$ deflection size as the HV detection threshold, despite the fact that an earlier HV study [25] emphasized that the $15^{\circ}$ value, which was the mean of radiologically measured 1stMTPJAs in adolescents, should not be used as a diagnostic HV criterion. Therefore, because they did not detect lateral overloadings with the $15^{\circ}$ threshold, they might not have detected a supination mechanism. For this reason, only size-dependent load characteristics might have been observed, i.e., central overloading with medial offloading. Additionally, because the medial
RFSP is reduced concomitantly with increases in the central RFSP, a medial RFSP reduction might be interpreted as a symptom of central overloading, or even a pronation-based mechanism, because the medial forefoot regions might be poorly visible and sometimes not detected at all. Recent studies that employed the radiological imaging method [24] could not confirm that the pronation mechanism was a distinguishing feature of $\mathrm{HV}$, because this mechanism was also commonly observed during loading in asymptomatic feet.

The findings on how the hallux alignment mediates weight-shifting by the foot represents a new avenue of research in HV pathology. Observation that the weight-shifting freedom by the foot was limited by the whole-body movement strategy might promote the development of new diagnostic approaches in HV pathology. Indeed, the apparent "otherness" of the limitation at the largest observed hallux deviation, compared to the limitations associated with any of the lesser deviations, might serve as a functional marker of HV risk. Thus, a disparity in the frequency of central and medial patterns of weight-shifting by the foot might serve as a functional criterion that sets the boundary between pathological and physiological states of hallux deflection. Maintaining or restoring physiological variety in performing weight-shifting by the foot during walking should be tested as a basis for HV therapies and for the prevention of $\mathrm{HV}$, in the early stages of pathology. The complexity of the phenomena involved in hallux alignment could explain the discrepant conclusions from previous studies on $\mathrm{HV}$ aetiology.

The individual tendency of using particular weight-shifting patterns should be taken into account, when creating new approaches in HV therapy. To date, HV treatments have focused exclusively on the affected part of the foot; these treatments include passive corrections or active strengthening of the toe muscles. Considering the way that long-term foot function contributes to shaping foot structure, a novel HV remedy could be to restore or maintain physiological variability in the whole-body movements performed in walking. For example, facilitating or forcing the execution of movements that are lacking could induce the appropriate variability in foot patterns [8]. This type of treatment might also improve balance and prevent falls associated with HV. Future longitudinal studies that include individuals with advanced deformities could facilitate the identification of cause-and-effect relationships of observed phenomena and the determination of their practical significance in therapeutic or prophylactic procedures in HV. 
The methodical research approach presented here allowed capturing motor control phenomena involved in hallux alignment, observable thanks to the new plantar pressures assessment - GMD method. This method is not yet quite automatic, which in practice limited the number of data. Nonetheless, findings of this study proved the concept of this method, justifying the start of design work towards its full automation, which would allow for the repeat of the research presented in the article on a larger number of participants or samples. Moreover, the framework adopted in the study: the GMD as a functional index, the frequency analysis in assessment of movement variability, and a BMI as an index of mechanical properties seems to be useful in studies on function - structure relationships.

\section{Conclusions}

Valgus alignment of hallux limits the freedom in realization of body weight-shifting through the plantar surface of the foot. Motor control processes should therefore be considered as an etiological factor of HV. Individuals with valgus alignment of the hallux favored or disfavored one weight-shifting pattern at the expense of another along two different sex-related pathways: girls displayed a relatively higher incidence of the lateral weight-shifting pattern, and boys displayed a relatively higher incidence of the medial weight-shifting pattern. Distinct limitations observed at $20^{\circ} 1$ stMTPJ angle in girls relied on the frequent use of a central, rather than medial, shift pattern and might serve as a criterion of $\mathrm{HV}$ pathology. Limitations in foot movements that affected natural gait variability seemed to result from a whole-body movement strategy for maintaining balance during walking.

\section{References}

[1] Agresti A., CAFFo B., Simple and effective confidence intervals for proportions and difference of proportions result from adding two successes and two failures, Am. Stat., 2000, 54 (4), 280-288.

[2] Baida S.R., Gore S.J., Franklyn-Miller A.D., Moran K.A., Does the amount of lower extremity movement variability differ between injured and uninjured populations? A systematic review, Scand. J. Med. Sci. Sports, 2018, 28 (4), 1320-1338.

[3] BlaKer H., Confidence curves and improved exact confidence intervals for discrete distributions, Can. J. Stat., 2000, 28 (4), 783-798.

[4] Choi Y.R., Lee H.S., Kim D.E., Lee D.H., Kim J.M., AhN J.Y., The diagnostic value of pedobarography, Orthopedics, 2014, 37 (12), e1063-e1067.
[5] Chopra S., Moerenhout K., Crevoisier X., Characterization of gait in female patients with moderate to severe hallux valgus deformity, Clin. Biomech., 2015, 30 (6), 629-635.

[6] Cliff N., Dominance statistics: Ordinal analyses to answer ordinal questions, Psychol. Bull., 1993, 114, 494-509.

[7] Davids K., Glazier P., AraúJo D., Bartlett R., Movement systems as dynamical systems: the functional role of variability and its implications for sports medicine, Sports Med., 2003, 33 (4), 245-460.

[8] Dhawale A.K., Smith M.A., Ölveczky B.P., The Role of Variability in Motor Learning, Annu. Rev. Neurosci., 2017, 40, 479-498.

[9] Franco P.S., Silva C.B., Rocha E.S., CARPeS F.P., Variability and repeatability analysis of plantar pressure during gait in older people, Rev. Bras. Reumatol., 2015, 55 (5), 427-433.

[10] Galica A.M., Hagedorn H.J., Dufour A.B., RiskowsKi J.L., Hillstrom H.J., CASEY V.A. et al., Hallux valgus and plantar pressure loading: the Framingham foot study, Foot Ankle Res., 2013, 6, 42.

[11] Gimunová M., Zvonař M., Mikeska O., The effect of aging and gender on plantar pressure distribution during the gait in elderly, Acta Bioeng. Biomech., 2018, 20, 139-144.

[12] Glasoe W.M., Treatment of Progressive First Metatarsophalangeal Hallux Valgus Deformity: A Biomechanically Based Muscle - Strengthening Approach, J. Orthop. Sports Phys. Ther., 2016, 46 (7), 596-605.

[13] Greve J., Alonso A., Bordini A.C., Camanho G.L., Correlation between body mass index and postural balance, Clinics., 2007, 62, 717-720.

[14] Hagedorn T.J., Dufour A.B., Riskowski J.L., Hillstrom H.J., Menz H.B., CASEY V.A. et al., Framingham Foot Disorders, Foot Posture, and Foot Function: The Framingham Foot Study, PLOS, 2013, 5, https://doi.org/10.1371/journal.pone.0074364

[15] HausdorfF J.M., Gait variability: methods, modeling and meaning, J. Neuroeng. Rehabil., 2005, 2, 19.

[16] Hiroto O., SAChiko J., Ai U., Tomohiro M., Masayuki S., Factors Related to Prevalence of Hallux Valgus in Female University Students: A Cross-Sectional Study, J. Epidemiol., 2014, 24 (3), 200-208.

[17] Janssen D.M., SANDERs A.P., Guldemond N.A., Hermus J., WALENKAMP G.H., VAN RHIJN L.W., A comparison of hallux valgus angles assessed with computerised plantar pressure measurements, clinical examination and radiography in patients with diabetes, J. Foot Ankle Res., 2014, 21, 7-33.

[18] LATASH M.L., The bliss (not the problem) of motor abundance (not redundancy), Exp. Brain Res., 2012, 217(1), 1-5.

[19] Latour E., Latour M., ARLET J., ADACH Z., BohatyrewicZ A., Gait functional assessment: Spatio-temporal analysis and classification of barefoot plantar pressure in a group of 1112-year-old children, Gait Posture, 2011, 34, 415-420.

[20] Meyers-Rice B., Sugars L., McPoil T., Cornwall M.W., Yavuz M., Hetherington V.J., Botek G., Hirschman G.B., BARDSLEY L., DAVIS B.L., Forefoot plantar shear stress distribution in hallux valgus patients, Gait Posture, 2009, 30 (2), 257-259.

[21] Michael F., Visualizing Categorical Data, SAS Institute, Cary, NC, 2000.

[22] Mickle K.J., Munro B.J., Lord S.R., Menz H.B., Steele J.R., Gait balance and plantar pressures in older people with toe deformities, Gait Posture, 2011, 34 (3), 347-51.

[23] Nguyen U.S., Hillstrom H.J., Li W., Dufour A.B., Kiel D.P., PROCTER-GRAY E. et al., Factors associated with hallux valgus in a population - based study of older women and men: 
the MOBILIZE Boston Study, Osteoarthritis Cartilage, 2010, 18 (1), 41-46.

[24] Ota T., NAGURA T., YAMAdA Y., YAMAdA M., YoKoyama Y., Ogihara N., Matsumoto M., NaKamura M., JinZAKi M., Effect of natural full weight - bearing during standing on the rotation of the first metatarsal bone, Clin. Anat., 2019, 32 (5), 715-721.

[25] Piggott H., The natural history of hallux valgus in adolescence in early adult life, J. Joint Bone Surg., 1960, 42, 749-760.

[26] Stergiou N., DeCKer L.M., Human movement variability, nonlinear dynamics, and pathology: is there a connection?, Hum. Mov. Sci., 2011, 30(5), 869-888.
[27] Tuna H., Yildiz M., Celtik C., Kokino S., Static and $d y$ namic plantar pressure measurements in adolescents, Acta Orthop. Traumatol. Turc., 2004, 38, 200-205.

[28] Van Emmerik R.E.A., Ducharme S.W., Amado A.C., HAMill J., Comparing dynamical systems concepts and techniques for biomechanical analysis, J. Sport Health Sci., 2016, 5 (1), 3-13.

[29] WaldeCKer U., Pedographic analysis of hallux valgus deformity, Foot Ankle Surg., 2004, 10, 121-124.

[30] Wen J., Ding Q., Yu Z., Sun W., Wang Q., Wei K., Adaptive changes of foot pressure in hallux valgus patients, Gait Posture, 2012, 36 (3), 344-349. 